\title{
Numerical Bifurcation Analysis of Lattice Boltzmann Models: A Reaction-Diffusion Example
}

\author{
Pieter Van Leemput and Kurt Lust \\ Department of Computer Science, K.U.Leuven, \\ Celestijnenlaan 200A, B-3001 Heverlee, Belgium. \\ \{pieter.vanleemput, kurt.lust\}@cs.kuleuven.ac.be
}

\begin{abstract}
We study two strategies to perform a time stepper based numerical bifurcation analysis of systems modeled by lattice Boltzmann methods, one using the lattice Boltzmann model as the time stepper and the other the coarse-grained time stepper proposed in Kevrekidis et al., CMS 1(4). We show that techniques developed for time stepper based numerical bifurcation analysis of partial differential equations (PDEs) can be used for lattice Boltzmann models as well. The results for both approaches are also compared with an equivalent PDE description.
\end{abstract}

Keywords: numerical bifurcation analysis, lattice Boltzmann method, Newton-Picard method, reaction-diffusion systems.

\section{Introduction}

Time simulation is a popular method to study the influence of varying the parameters in a parameter-dependent dynamical system, but comparing simulation results for many different parameter values is tedious. Also, only stable solutions can be explored in this way. Bifurcation theory offers a more systematic way to study the asymptotic behavior of a parameter-dependent system.

In a numerical bifurcation analysis, one computes branches of solutions of some type by varying one or more parameters. Along the branch, the stability information is monitored and the critical (or bifurcation) points where stability changes occur are detected and often computed. Several techniques and codes have been developed for partial differential equations (PDEs), some of which work on top of an existing time simulation code. In this paper, we show that these techniques can be used for bifurcation analysis of lattice Boltzmann (LB) models also. Examples of such time stepper or map based techniques are the Recursive Projection Method [1] and the Newton-Picard method [2]. These methods exploit a spectral property of the time integration operator that is typical of systems with a dissipative operator: the time integration operator has only few eigenvalues close to or outside the unit circle. In other words, the system's long-term behavior is governed by only a small number of slow modes. This is an 
inherent property of the physics of the system. It is also the starting assumption for techniques such as inertial manifolds and reduced-order modeling.

We will discuss two strategies for the bifurcation analysis of LB models. One approach uses the LB time stepper as the time stepper in the bifurcation code. The state can be expressed either as distribution functions or as a full set of velocity moments. We will also make a slight extension of the time stepper to compute periodic solutions. The second approach uses the coarse-grained time stepper proposed by Kevrekidis et al. [34] which performs a time integration for the macroscopic variables (a subset of the velocity moments in a LB model) using only microscopic or mesoscopic simulations. Their coarse-grained time stepper is developed for cases where a macroscopic model is conceptually possible, but not yet known in a closed analytical form. Whereas our numerical bifurcation analysis techniques rely on a separation of time scales at the macroscopic level, the coarse-grained time stepper relies on a time scale separation between the macroscopically relevant variables, i.e., the lower-order moments of the distribution functions in a LB model, and the other variables that are only relevant at the microscopic or mesoscopic scales.

We will use a FitzHugh-Nagumo (FHN) reaction-diffusion system on a onedimensional domain as our test case. This system is usually specified as a PDE system, but it is possible to develop an equivalent LB-BGK model. We compare the results for both approaches based on the LB model with each other and with the results for the equivalent PDE model. Section 2 focusses on the numerical bifurcation analysis. In Sect. 3, we present the three models. Numerical results for the FHN system are shown in Sect. 4. Section 5 repeats the main conclusions.

\section{Numerical Bifurcation Analysis}

For numerical bifurcation analysis of autonomous parameter-dependent PDEs, the PDEs are first space-discretized to a large set of ordinary differential equations (ODEs)

$$
y_{t}=f(y, \gamma), \quad f: \mathbb{R}^{N} \times \mathbb{R} \mapsto \mathbb{R}^{N}
$$

with $y$ the state vector and $\gamma$ the bifurcation parameter. Let $\varphi_{T}(y(0), \gamma)$ denote the result $y(T)$ of a time integration of (1) at time $T$ with initial condition $y(0)$ and parameter $\gamma$. A steady state $\left(y^{*}, \gamma^{*}\right)$ of (1) is also a fixed point of the map

$$
y \mapsto \varphi_{T}(y, \gamma)
$$

for any value of $T$. A periodic solution of (11) is a fixed point of (2) only when $T$ is a multiple of the (unknown) period.

A steady state of (10) is asymptotically stable if all eigenvalues $\lambda_{j}$ of the Jacobian matrix $(\partial f / \partial y)\left(y^{*}, \gamma^{*}\right)$ have a negative real part. Hence the rightmost eigenvalues determine the asymptotic stability. The equivalent fixed point of (2) is stable if all eigenvalues $\mu_{j}$ of the matrix $M:=\left(\partial \varphi_{T} / \partial y\right)\left(y^{*}, \gamma^{*}\right)$ are smaller than one in modulus. Both sets of eigenvalues are related by

$$
\mu_{j}=\exp \left(\lambda_{j} T\right)
$$


and thus $\left|\mu_{j}\right|<1 \Leftrightarrow \operatorname{Re}\left(\lambda_{j}\right)<0$. Hence they provide exactly the same stability information.

In time stepper based bifurcation analysis, a continuous-time dynamical system is analyzed through the (almost) equivalent map (2). In fact, the time integrator can be any time integrator, including a LB simulation or the coarsegrained time integrator. A branch of fixed points is computed with a continuation method. Given a few points on the branch, a prediction is made for the next point and that point is then computed by solving the nonlinear system

$$
\left\{\begin{array}{l}
\varphi_{T}(y, \gamma)-y=0 \\
n(y, \gamma, \eta)=0
\end{array}\right.
$$

for $y$ and $\gamma$. The scalar parameterizing equation $n(y, \gamma, \eta)=0$ determines the position of the point along the branch through a reparameterization with parameter $\eta$. In our experiments, we used pseudo-arclength parameterization 5 .

When computing a branch of periodic solutions, the period $T$ becomes a variable as well. The system (4) is augmented with a phase condition $s(y, T, \gamma)=0$, which ensures isolated solutions of the system. $M$ is then called the monodromy matrix and its stability-determining eigenvalues the Floquet multipliers.

A popular way of solving (41) is Newton's method with Gaussian elimination. However, $M$ and thus also the Jacobian matrix of (4) are in general dense matrices (even though $(\partial f / \partial y)(y, \gamma)$ is usually a sparse matrix). It is not feasible to compute and store $M$. However, since computing a matrix-vector product with $M$ is equivalent to computing a directional derivative of the time integrator, these matrix-vector products can be computed using numerical differentiation.

The fact that $M$ often has only a few eigenvalues close to or outside the unit circle is exploited by several numerical methods. One of the earliest is the Recursive Projection Method [1]. Although this method has only been derived for the computation of steady state solutions, it can be extended to compute periodic solutions also. However, robustness problems are fairly common with the original implementation of [1]. We will use the more robust Newton-Picard method [2] instead. This method was originally developed for the computation of periodic solutions but is equally well suited for the computation of steady states. First, an approximation to the low-dimensional generalized eigenspace $\mathcal{U}$ of all dominant eigenvalues of $M$ is computed by orthogonal subspace iterations, requiring only matrix-vector products with $M$. Then, the linearized system is projected onto $\mathcal{U}$ and its high-dimensional orthogonal complement $\mathcal{U}^{\perp}$. The large subsystem in $\mathcal{U}^{\perp}$ is solved approximately using a fixed point (or Picard) iteration, which corresponds essentially to time integration in the high-dimensional subspace $\mathcal{U}^{\perp}$. The small subsystem in $\mathcal{U}$ is solved using Gaussian elimination. The total number of time integrations needed to converge to a given accuracy is mostly determined by the dominant eigenvalues and thus by the physics of the problem and not by the particular mathematical model or discretization. Hence we expect that a similar number of time integrations will be needed for the PDE model, the LB model and the coarse-grained description, though some differen- 
ces are possible, e.g., because of a different number of variables or convergence problems of Newton's method when the nonlinearity becomes too strong.

\section{System Descriptions}

\subsection{Macroscopic Description: A PDE System}

The one-dimensional macroscopic FHN PDE model is given by

$$
\left\{\begin{array}{l}
\rho_{t}^{a c}=\rho_{x x}^{a c}+\rho^{a c}-\left(\rho^{a c}\right)^{3}-\rho^{i n}, \\
\rho_{t}^{i n}=\delta \rho_{x x}^{i n}+\varepsilon\left(\rho^{a c}-a_{1} \rho^{i n}-a_{0}\right),
\end{array}\right.
$$

with homogeneous Neumann boundary conditions on the domain $[0,20]$. The variables $\rho^{a c}(x, t)$ and $\rho^{i n}(x, t)$ are the activator and inhibitor concentration respectively. We set $\delta=4, a_{0}=-0.03$ and $a_{1}=2$ and vary $\varepsilon \in[0,1]$. We used a method of lines discretization with central differences in space at the midpoints of 200 lattice intervals and the trapezoidal rule for time integration.

\subsection{Mesoscopic Description: Lattice Boltzmann Model}

The variables in a LB model are the distribution functions $f_{i}^{s}(x, t)$ associated with a species $s \in\{a c, i n\}$ and a discrete "particle" velocity $v_{i}$. They are defined on a space-time lattice with spacing $\Delta x$ in space and $\Delta t$ in time. We use only three discrete values for the velocity: $v_{i}=i \Delta x / \Delta t$ with $i \in I:=\{-1,0,1\}$ (D1Q3 scheme). The concentration - the only relevant macroscopic variable - is the zeroth order velocity moment of the distribution functions, i.e.,

$$
\rho^{s}(x, t)=\sum_{i=-1}^{1} f_{i}^{s}(x, t) .
$$

The discrete time evolution of the distribution functions is described by the standard LB time stepper equation

$$
f_{i}^{s}\left(x+v_{i} \Delta t, t+\Delta t\right)-f_{i}^{s}(x, t)=-\omega^{s}\left[f_{i}^{s}(x, t)-f_{i}^{s, e q}(x, t)\right]+R_{i}^{s}, i \in I .
$$

We used halfway bounce-back boundary conditions [6] and the approach in [7] for the BGK collision term $-\omega^{s}\left[f_{i}^{s}(x, t)-f_{i}^{s, e q}(x, t)\right]$ and reaction term $R_{i}^{s}$. Note that the equilibrium distribution $f_{i}^{s, e q}(x, t)=\frac{1}{3} \rho^{s}(x, t), i \in I$ which will be important in the remaining of the paper.

Note that the single local time scale in the LB model should not be confused with the global time scales which are important in our numerical algorithms. The large range of global time scales results from the coupling of all lattice points. The slowest components in the global behavior of the system can be much slower than the local relaxation time.

When computing periodic solutions, the integration time $T$ is continuous and not always an exact multiple of the LB time step $\Delta t$. We then determine a positive integer $k$ such that $(k-1) \Delta t<T \leq k \Delta t$ and compute the state at time $T$ by a linear interpolation of the states at the discrete time steps $k-1$ and $k$. 


\subsection{Coarse-Grained Description}

The coarse-grained LB (CGLB) time integration procedure proposed in [34] is a time stepper for the macroscopic variables. A macroscopic time step $\Delta T$ consists of three basic steps. The first step is the reconstruction or lifting. Here, meaningful mesoscopic initial values $f_{i}^{s}(x, 0)$ are derived, which are consistent with the governing macroscopic variable $\rho^{s}(x, 0)$, i.e.,

$$
f_{i}^{s}(x, 0)=w_{i} \rho^{s}(x, 0), i \in I \quad \text { with } \quad \sum_{i=-1}^{1} w_{i}=1 .
$$

We choose the weights $w_{i}$ all equal to $1 / 3$, i.e., equal to the weights for the diffusive equilibrium. Next, the mesoscopic variables $f_{i}^{s}(x, t)$ are evolved through the LB time stepper for a time interval $\Delta T$. If $\Delta T$ is not an integer multiple of the LB time step $\Delta t$, we interpolate as in Sect. 3.2. In the final restriction or coarse-graining step, the macroscopic variable $\rho^{s}(x, \Delta T)$ is computed using (6). This procedure is repeated until time $T$. In our experiments, we use $\Delta T=5$. Since $\Delta T$ is fairly large, we cannot interpolate between two time steps to obtain the result at an arbitrary time $T$. Instead we change $\Delta T$ slightly from the target value $\Delta T=5$, such that it fits an integer number of times in $T$. As long as $\Delta T$ is not changed too much, this has no significant influence on the result. The choice of the reconstruction weights $w_{i}$ and macroscopic time step $\Delta T$ is a topic of our current research and will be discussed in a future publication.

\section{Numerical Results and Discussion}

\subsection{Bifurcation Diagrams}

Figure 1 shows the bifurcation diagram for steady state (upper diagram) and periodic solutions (lower diagram), computed using the Newton-Picard method. We used $T=5$ for the steady state solutions, a good value for the Newton-Picard method in this case. For the LB model, we used $\Delta t=0.001$, which produced the best results. The steady state branch contains two bifurcation points: a fold point at $\varepsilon \approx 0.945$ and a supercritical Hopf point at $\varepsilon \approx 0.0183$ where the branch of periodic solutions in the lower diagram meets. The periodic solution branch has a fold point at $\varepsilon \approx 0.00087$. Though the unstable part of the branch has almost the same $(\varepsilon, T)$-projection as the stable one, the corresponding orbits are different. Computing these unstable solutions proved difficult, which is a wellknown problem of single shooting based numerical methods. The bifurcation diagrams for the two LB approaches correspond very well with differences on the order of the discretization error. Moreover, we do obtain the same bifurcation information as from the equivalent PDE.

\subsection{Eigenvalue Spectra}

The Newton-Picard code computes the stability-determining eigenvalues through orthogonal subspace iterations. Table 1 lists the dominant eigenvalues $\lambda_{j}$ for an 

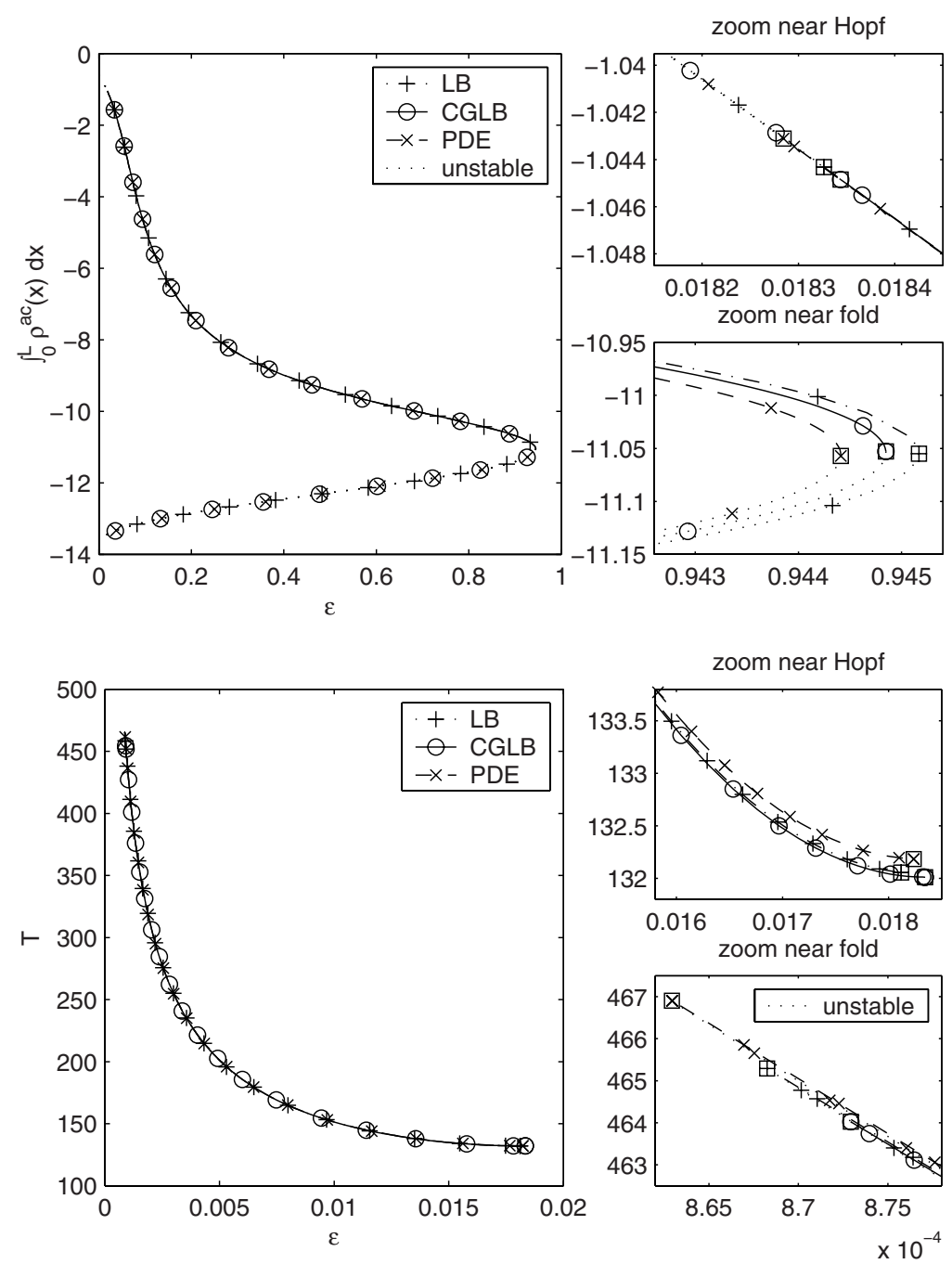

Fig. 1. Bifurcation diagram for the steady state (upper diagram) and periodic solutions. Unstable solutions are indicated by dotted lines and bifurcation points by boxed markers. The markers represent only a subset of the computed points

Table 1. Dominant eigenvalues for the unstable steady state on the upper part of the branch and stable periodic solution at $\varepsilon=0.01$ (using $\Delta T \approx 5$ in the CGLB integrator)

\begin{tabular}{|l||c|c|c||c|c|}
\hline \multicolumn{1}{|c||}{} & \multicolumn{3}{c||}{ steady state } & \multicolumn{2}{c|}{ periodic solution } \\
\cline { 2 - 6 } & $\lambda_{1,2}$ & $\lambda_{3}$ & $\lambda_{4}$ & trivial $\mu_{1}$ & $\mu_{2}$ \\
\hline \hline LB & $0.002010 \pm 0.039461 i$ & -0.124867 & -0.411364 & 1.000000 & 0.514888 \\
\hline CGLB & $0.002012 \pm 0.039463 i$ & -0.124863 & -0.411348 & 1.000000 & 0.514452 \\
\hline PDE & $0.001999 \pm 0.039446 i$ & -0.124861 & -0.411288 & 1.000000 & 0.516712 \\
\hline
\end{tabular}


unstable steady state at $\varepsilon=0.01$ and Floquet multipliers $\mu_{j}$ for the stable periodic solution at the same parameter value. Again, the eigenvalues for both LB based approaches correspond very well with one another and give the same information as the equivalent PDE model. Note that periodic solutions of an autonomous system always have a trivial multiplier at one. The great accuracy of the computed value indicates that the time integration and eigenvalue computation is very accurate.

\subsection{Time Stepper Calls}

Table 2 lists the number of time integrations needed to continue a part of the branches of stable steady state and stable periodic solutions, not including the integrations for the accurate computation of the stability information at the end. For the steady states, we again used $T=5$. Note that the LB model uses a different set of variables from the other two approaches and hence another measure for the distance. We changed the maximal step size in our variable step size continuation code for each approach such that the number of points computed along the branch is approximately the same. As expected, the average number of time integrations needed for each point is almost the same. The computing time however is much lower for the PDE based model since the implicit time stepper uses much larger steps than the LB model. The computing time for both LB based approaches is dominated by the LB simulations and virtually the same for both approaches.

Table 2. Total number of time integrations, the number of points computed and the average number of time integrations for the continuation of a part of the branches of stable steady state and periodic solutions

\begin{tabular}{|l||c|c|c||c|c|c|}
\hline \multirow{2}{*}{$\begin{array}{l}\text { number of time } \\
\text { integrations }\end{array}$} & \multicolumn{3}{|c||}{ steady state, $\varepsilon \in[0.05,0.55]$} & \multicolumn{3}{c|}{ periodic, $\varepsilon \in[0.006,0.016]$} \\
\cline { 2 - 7 } & total & nb. pts & avg. & total & nb. pts & avg. \\
\hline \hline LB & 1352 & 20 & 67.600 & 1611 & 25 & 64.440 \\
\hline CGLB $(\Delta T \approx 5)$ & 1419 & 21 & 67.571 & 1623 & 25 & 64.920 \\
\hline PDE & 1420 & 21 & 67.619 & 1621 & 25 & 64.840 \\
\hline
\end{tabular}

\section{Conclusions}

In this paper, we have shown that algorithms developed for time stepper based numerical bifurcation analysis of PDEs can be used for lattice Boltzmann models as well in two different ways. It is possible to use either the LB model itself or the coarse-grained time integrator as the time stepper in a time stepper based bifurcation code. For our test case, the accuracy and computing time for both LB based approaches are comparable which was shown to be not unexpected. We 
have also compared the results to those for an equivalent PDE model, demonstrating that the same bifurcation information can be obtained from a lattice Boltzmann model as from a PDE model. However, time integration of the PDE was much faster, resulting in much lower computing times for the bifurcation diagram of the PDE model.

The two approaches for bifurcation analysis of LB models have distinct advantages and disadvantages. Several parameters in the coarse-grained integrator need to be chosen carefully. This is currently a subject of our research and will be discussed in a future publication. On the other hand, the dimension of the state vector is much lower (only one third of the number of components in our case), resulting in a proportionally lower memory consumption of the NewtonPicard method for the coarse-grained time integrator. In some cases, this can be an issue and thus possibly an advantage of the coarse-grained approach.

Acknowledgements. Kurt Lust is a postdoctoral fellow of the Fund for Scientific Research - Flanders which also provided further funding through project G.0130.03. This paper presents research results of the Interuniversity Attraction Poles Programme - Belgian Science Policy. The scientific responsibility rests with its authors. We also acknowledge many helpful discussions with Prof. I.G. Kevrekidis.

\section{References}

1. Shroff, G.M., Keller, H.B.: Stabilization of unstable procedures: The Recursive Projection Method. SIAM Journal on Numerical Analysis 30 (1993) 1099-1120

2. Lust, K., Roose, D., Spence, A., Champneys, A.: An adaptive Newton-Picard algorithm with subspace iteration for computing periodic solutions. SIAM Journal on Scientific Computing 19 (1998) 1188-1209

3. Kevrekidis, I.G., Gear, C.W., Hyman, J.M., Kevrekidis, P.G., Runborg, O., Theodoropoulos, C.: Equation-free, coarse-grained multiscale computation: Enabling microscopic simulators to perform system-level analysis. Communications in Mathematical Sciences 1 (2003) 715-762

4. Theodoropoulos, C., Qian, Y.H., Kevrekidis, I.G.: "Coarse" stability and bifurcation analysis using time-steppers: a reaction-diffusion example. Proceedings of the National Academy of Sciences 97 (2000) 9840-9843

5. Keller, H.B.: Numerical solution of bifurcation and nonlinear eigenvalue problems. In Rabinowitz, P.H., ed.: Applications of Bifurcation Theory, New York, Academic Press (1977)

6. Ginzbourg, I., Adler, P.M.: Boundary flow condition analysis for the three-dimensional lattice Boltzmann model. Journal of Physics II France 4 (1994) 191-214

7. Qian, Y.H., Orszag, S.A.: Scalings in diffusion-driven reaction $A+B \rightarrow C$ : Numerical simulations by Lattice BGK Models. Journal of Statistical Physics $\mathbf{8 1}$ (1995) 237-253 